\title{
Epidemiology of reported Yersinia enterocolitica infections in Germany, 2001-2008
}

\author{
Bettina M Rosner*, Klaus Stark and Dirk Werber
}

\begin{abstract}
Background: Yersiniosis is the third most common zoonotic bacterial disease in Germany and the European Union. Sequelae of Yersinia enterocolitica infections, such as reactive arthritis, have been reported. Consumption of pork and its products, especially eaten raw or undercooked, is an important risk factor of yersiniosis. Infection with Y. enterocolitica is notifiable through the national surveillance system for infectious diseases in Germany and several thousands of cases are being reported each year. We present recent data on the epidemiology of reported yersiniosis in Germany.

Methods: Surveillance data on yersiniosis, accessed through the national level database (SurvNet), were analyzed with regard to time trends, demographical and geographical distribution, serotypes, and hospitalization, for the time period 2001-2008.

Results: A total of 47,627 cases of yersiniosis were reported. The mean annual incidence of yersiniosis was 7.2/100,000 population. A downward trend in the number of reportable cases has occurred since 2002. Almost all Y. enterocolitica infections were reported as single cases, i.e., with no apparent links to other cases. The number of reported infections showed substantially less seasonal variation than in other zoonotic enteric diseases. The incidence was highest in children under five years (58/100,000 population), in particular in one-year-old children (108/100,000 population). Almost $97 \%$ of infections were acquired domestically. High incidences occurred in the eastern German federal states Thuringia, Saxony, and Saxony-Anhalt. Differences in incidences across federal states were driven primarily by incidence differences in children under five years. Hospitalization was reported for $17 \%$ of cases, the proportion being highest among teenagers. Almost $90 \%$ of Y. enterocolitica strains were diagnosed as serotype $0: 3$, which is the serotype most frequently isolated from pigs.

Conclusions: Yersiniosis is a zoonotic foodborne disease of relevance to public health in Germany because of its high incidence and risk for sequelae. The incidence of reported yersiniosis in Germany varies markedly from state to state, mainly due to incidence difference among young children. More research efforts should be directed towards the elucidation of risk factors of yersiniosis in this age group.
\end{abstract}

\section{Background}

Yersiniosis due to infection with the bacterium Yersinia enterocolitica is a zoonotic gastrointestinal disease in humans. Y. enterocolitica species can be isolated from a variety of domestic and wildlife animals, e.g., pigs, cattle, sheep, goats, dogs, cats, wild boars, and small rodents [1]. Pigs are considered to be the main reservoir of human pathogenic strains, largely because of the high prevalence of these strains in pigs and the high genetic similarity between porcine and human isolates [2-4]. Infections are

* Correspondence: RosnerB@rki.de

1 Robert Koch Institute, Department of Infectious Disease Epidemiology, DGZRing 1, 13086 Berlin, Germany

Full list of author information is available at the end of the article thought to be primarily transmitted to humans by food, in particular, raw or undercooked pork and pork products $[1,5]$. However, other risk factors, such as contaminated drinking water or pet animal contact, have been reported [6-9]. Six different biotypes (biotype 1A, 1B, 25 ) and numerous serotypes of $Y$. enterocolitica have been described. Eleven of those serotypes have frequently been associated with infections in humans [1]. In Europe, most of the human pathogenic $Y$. enterocolitica strains are classified as biotype 4, serotype O:3 [10]. Clinical symptoms of yersiniosis first appear after an incubation period of about 5 days (range 1-11 days) and include diarrhea, fever, vomiting, tenesma and abdominal pain. In older children and young adults, abdominal pain in the right 
lower abdomen can occur, which may be mistaken for appendicitis (pseudoappendicitis). Typically, symptoms disappear within 1-2 weeks after onset. Sequelae such as reactive arthritis or erythema nodosum sometimes occur [11]. Yersiniosis contributes substantially to foodborne diseases in industrialized countries and is therefore notifiable through national surveillance systems in most countries within the European Union (EU), including Germany. After campylobacteriosis and salmonellosis, yersiniosis ranks third among the notifiable bacterial zoonoses in Germany and the EU. In 2007, 8,874 confirmed cases of yersiniosis were reported to the European Centre for Disease Control and Prevention (ECDC), 4,987 (56\%) of which were from Germany [12]. There is a paucity of recent comprehensive data on the epidemiology of yersiniosis, e.g., detailed analysis of trends over time or affected population groups.

\section{Methods}

Yersiniosis surveillance data from Germany were analyzed for the time period 2001-2008. An acute cultureconfirmed infection with Yersinia enterocolitica is notifiable to the local health department according to the Protection against Infection Act (Infektionsschutzgesetz, IfSG) of 2001. Each notification has to be electronically forwarded from the local health department via the state health department to the federal public health institute, the Robert Koch Institute (RKI), where the national surveillance database is hosted. To ensure comparability of surveillance data across federal states, surveillance case definitions exist for each notifiable condition. A case of yersiniosis is included in the RKI statistics when the diseased person being reported as a case showed clinical symptoms (i.e. at least one of the following: diarrhea, abdominal pain, tenesma, fever with body temperature of $38.5^{\circ} \mathrm{C}$ or above, and vomiting) and the $Y$. enterocolitica infection was either culture-confirmed from stool or some other clinical material, or confirmed epidemiologically. Epidemiological confirmation of a case is defined as contact with another laboratory-confirmed case, contact with an animal infected with $Y$. enterocolitica, or consumption of food items contaminated with $Y$. enterocolitica. Prior to 2004, patients with clinical symptoms and serological evidence of infection (agglutination reaction (Widal), confirmation of IgA-, IgG- or IgM-antibodies by ELISA or Western blot) also fulfilled the case definition. Data were accessed through the national level database (SurvNet) at the RKI and analyzed with Microsoft Excel. Data are openly available via SurvStat@RKI http:// www3.rki.de/SurvStat/[13,14].

\section{Results}

\section{Time trend}

The total number of yersiniosis cases reported in the time period 2001-2008 was 47,627. The annual number ranged from 4,354 to 7,540 (Table 1), corresponding to an annual incidence of 5.3 to 9.2 infections per 100,000 population, with an average annual incidence of 7.2 infections per 100,000 population. Of all the reported Yersinia enterocolitica infections, $99.3 \%$ had been laboratory-confirmed and $0.7 \%$ had been confirmed epidemiologically. Of the laboratory-confirmed infections, 92\% had been cultureconfirmed. A downward trend in the annual number of reported $Y$. enterocolitica cases and, correspondingly, in the incidence has occurred since 2002 across all age groups and all German federal states, with an overall decrease of $42 \%$ in reported cases from 2002 to 2008 (Table 1). In contrast to other important zoonotic enteric diseases, e.g., those caused by Salmonella spp. or Campylobacter spp., which typically peak during the summer months, the seasonal distribution of reported $Y$. enterocolitica infections was relatively uniform, with only a slight increase in June, July, and September. The lowest number of $Y$. enterocolitica infections was reported in March and April (Figure 1). The majority of infections with Y. enterocolitica (about 98\%) was reported as single cases, i.e., with no apparent links to other cases. Over the study period, 19 to 53 clusters of yersinioses that affected a total of 40156 persons were reported to the RKI each year. Most clusters consisted of just 2 cases, typically from the same household. Clusters with $\geq 5$ epidemiologically related cases were reported only once or twice a year (data not shown).

\section{Demographic distribution}

$Y$. enterocolitica infections occurred more frequently in boys and men than in girls and women, with mean annual incidences of $8.0 / 100,000$ population and 6.5/100,000 population, respectively. Children were more frequently affected than adults. The average annual incidence of $Y$. enterocolitica infections in children $<5$ years of age was about 12-fold higher than the average in the German population aged $\geq 5(57.6 / 100,000$ population vs. 4.9 /

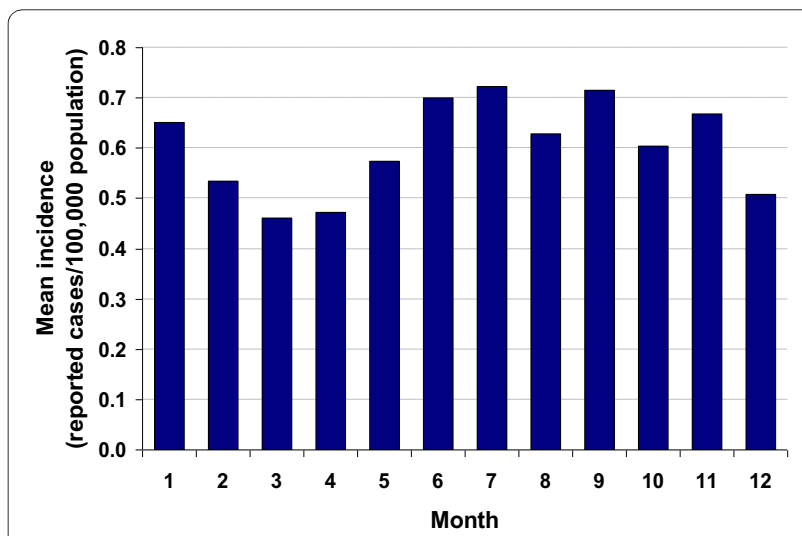

Figure 1 Seasonal distribution of reported Yersinia enterocolitica infections in Germany, 2001-2008. Data shows mean monthly incidence per 100,000 population. 
Table 1: Annual number of reported Yersinia enterocolitica infections in Germany according to serotype

\begin{tabular}{|c|c|c|c|c|c|c|c|c|}
\hline & \multicolumn{8}{|l|}{ Year } \\
\hline & 2001 & 2002 & 2003 & 2004 & 2005 & 2006 & 2007 & 2008 \\
\hline O:3 & 4304 & 5227 & 4826 & 4672 & 4306 & 3945 & 3935 & 3361 \\
\hline O:9 & 279 & 325 & 269 & 325 & 282 & 256 & 264 & 277 \\
\hline $0: 5,27$ & 32 & 29 & 35 & 44 & 48 & 59 & 35 & 30 \\
\hline O:8 & 1 & 0 & 0 & 5 & 14 & 16 & 8 & 18 \\
\hline Other & 175 & 342 & 432 & 185 & 149 & 155 & 118 & 145 \\
\hline No information on serotype & 2404 & 1617 & 1015 & 953 & 829 & 730 & 628 & 523 \\
\hline Total & 7195 & 7540 & 6577 & 6184 & 5628 & 5161 & 4988 & 4354 \\
\hline
\end{tabular}

100,000 population, respectively). The highest incidence of reported $Y$. enterocolitica infections occurred among one-year-old children (107.9/100,000 population) (Figure 2).

\section{Geographical distribution}

Each year, about $97 \%$ of reported yersiniosis infections with available information on the most likely country of infection were acquired domestically (Table 2). The mean annual incidence of reported $Y$. enterocolitica infections varied across the 16 German federal states, being highest in the eastern states Thuringia (23/100,000 population), Saxony (17/100,000 population), and Saxony-Anhalt (17/ 100,000 population), and lowest in the southern states Bavaria (5/100,000 population) and Baden-Wuerttemberg (3/100,000 population) (Figure 3). High overall annual incidences in federal states were mainly due to high incidences among children. Across federal states, mean annual incidences among children $<5$ years of age differed by a factor of almost 30 (e.g., Thuringia: 413/ 100,000 population, Baden-Wuerttemberg: 14/100,000 population). In comparison, incidences among adults ( $\geq 18$ years) differed only by a factor of about 3 across federal states (e.g., Mecklenburg-Western Pomerania: 6/ 100,000 population, Baden-Wuerttemberg: 2/100,000 population). Over the period of analysis, the number of reported yersinioses decreased in all federal states.

\section{Serotypes}

By far the most commonly reported human pathogenic $Y$. enterocolitica serotype was O:3 (Table 1). Of all notified cases with data on serotype, $89 \%$ were attributed to serotype $\mathrm{O}: 3,6 \%$ to serotype $\mathrm{O}: 9,0.8 \%$ to serotype $\mathrm{O}: 5,27$, $0.2 \%$ to serotype $\mathrm{O}: 8$, and $4 \%$ to other, non-specified serotypes. The number of reported cases with no available information on serotype decreased annually from about $33 \%$ in 2001 to $12 \%$ in 2008 (Table 1). The distribution of serotypes among reported cases varied according to age group. Over $90 \%$ of $Y$. enterocolitica infections among persons $<20$ years of age were caused by serotype O:3, compared with $70 \%$ among patients $\geq 60$. The proportion of infections caused by serotype 0:9 was higher in adults $\geq 40$ years of age than in younger patients (Table 3 ).

The causative agent was classified as $Y$. enterocolitica serotype O:3 in $89 \%$ of reported infections that were acquired in Germany or other countries of the EU and the European Free Trade Association (EFTA: EU plus Iceland, Liechtenstein, Norway, Switzerland). This percentage was only $77 \%$ when $Y$. enterocolitica infections were acquired in non-European countries. $Y$. enterocolitica serotype O:8 infections were more frequently imported from non-European countries than infections due to other serotypes (Table 2).

\section{Clinical aspects}

Diarrhea and abdominal pain were the most common symptoms of reported $Y$. enterocolitica infections (Table 4). Compared to other serotypes, abdominal pain and fever were reported less frequently ( 34 and $11 \%$, respectively), but diarrhea, vomiting and tenesma were reported more frequently $(94,10$, and $3 \%$, respectively) with $\mathrm{O}: 8$ infections. The percentage of patients with $Y$. enterocolitica infection for which a hospital stay was reported remained stable at about $17 \%$ throughout the observed time period regardless of diagnosed serotype, with the exception of serotype 0:8 (with 39\% of patients being hospitalized). The proportion of hospitalized patients varied with age, being highest for teenagers (10-19 years; $28 \%$ hospitalized) and elderly patients ( $\geq 60$ years of age; $25 \%$ hospitalized). The median length of stay in the hospital was 4 days, but this varied with sex, age group, and serotype. It became prolonged in patients that were female, $\geq 20$ years of age, and when isolates belonged to 


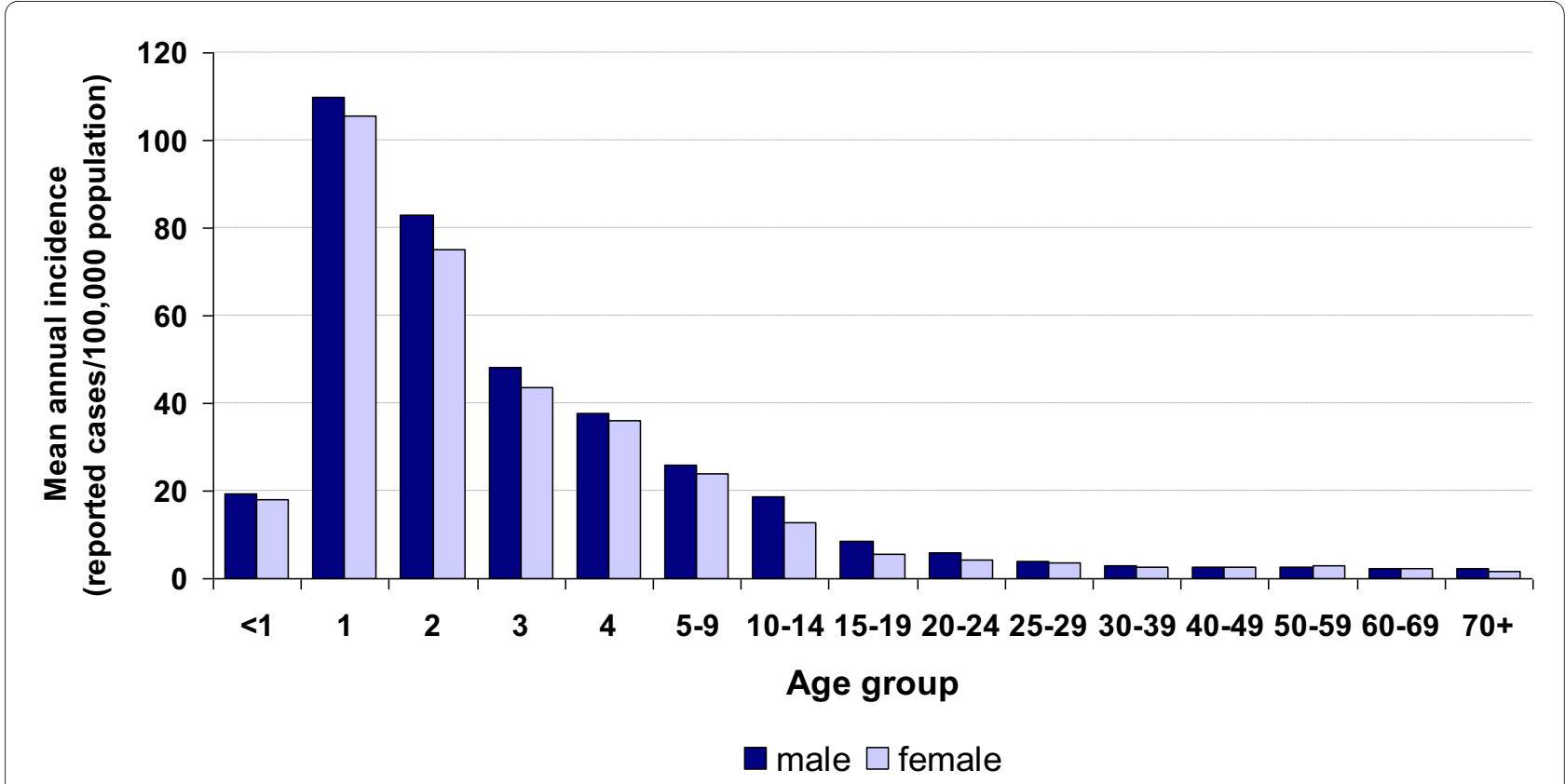

Figure 2 Reported Yersinia enterocolitica infections in Germany by age group and sex, 2001-2008. Data shows mean annual incidence per 100,000 population. Note that age group ranges vary.

serotypes other than O:3. The hospitalization rate was highest (34\%) and the median length of hospital stay was 8 days, when the pathogen was of serotype O:8. Between 2001 and 2008, 14 deaths due to Y. enterocolitica infection were reported to the national surveillance database. Six of the deceased persons were over 60 years. Serotypes of the $Y$. enterocolitica isolates were reported for 7 of the 14 deaths and included O:3 (5 deaths) and O:9 (2 deaths).

\section{Discussion}

German surveillance data on yersiniosis due to Yersinia enterocolitica infection for the time period 2001-2008 has been analyzed. The incidence of $Y$. enterocolitica infection was highest among children under 5 years of age, in particular one-year-old children. In addition to consumption of pork, other risk factors of yersiniosis need to be considered for this age group. A recent case-control study conducted in Sweden among children $<7$ years of age identified, in addition to pork consumption, contact to domestic animals, in particular dogs and cats, and use of a pacifier as risk factors [8]. Age-specific risk factors, with evidence of environmental transmission, have also been demonstrated in infections with Shiga toxin-producing $E$. coli (STEC) and Campylobacter spp. [15,16]. A high incidence of yersiniosis among young children has been reported in other countries, and acute diarrheal illnesses caused by enteric pathogens other than $Y$. enterocolitica, e.g., infections with Salmonella spp., Campylobacter spp., or E. coli including STEC, also occur at high frequencies among infants [17-20]. Factors that could contribute to the high incidence in reported diarrheal illnesses in this age group may include an increased rate of exposure to enteric pathogens as a result of fecal-oral contamination [21], predisposition to infection due to an immature and unchallenged immune system [21], higher frequency of physician consultations among parents of infants [22], or higher frequency of submission of stool samples for diagnosis by physicians when infants have been affected.

Within Germany, the highest incidences of yersiniosis occurred in the federal states Thuringia, Saxony, and Saxony-Anhalt. Similar results were obtained in a joint spatial analysis of 4 gastrointestinal infectious diseases, including yersiniosis, that took underreporting into account [23]. Although drawing causal inferences from group level data to the individual level is error-prone, it is interesting to note that, according to a recent national nutrition study, consumption of meat products and sausages was relatively high in the states with the highest incidences of yersiniosis (e.g. Saxony and Thuringia). Unfortunately, published data do not specify the type of meat consumed, making it unclear whether these results are also true for pork products [24]. The number of reported $Y$. enterocolitica infections did not show seasonal variability, which may support the hypothesis that the infection is transmitted via food items that are consumed consistently throughout the year, such as meat and meat products [24], rather than being associated with unknown environmental factors. Besides, environmental transmission of zoonotic enteric pathogens, direct contact to animals, for example, seems to be more pro- 


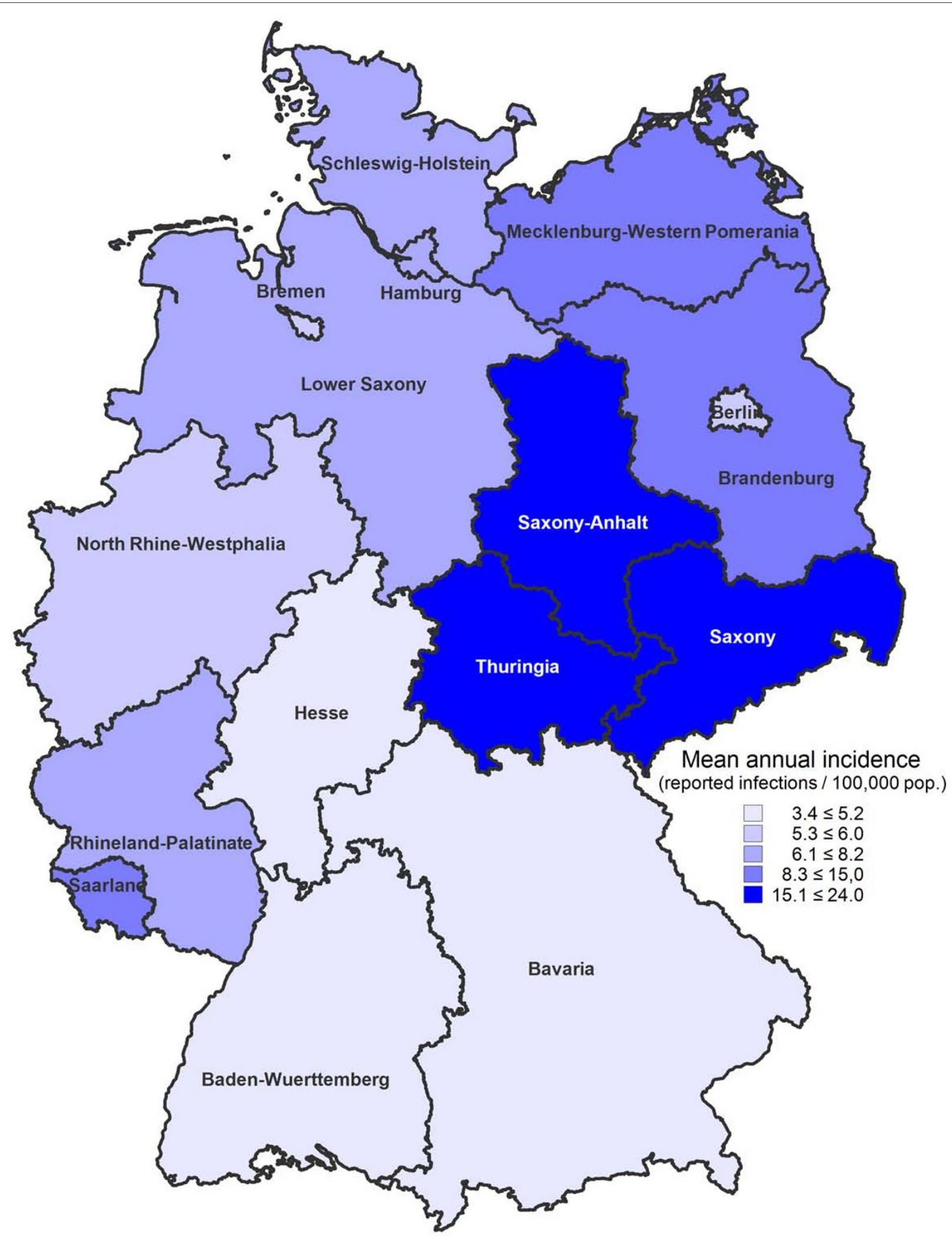

Figure 3 Reported Yersinia enterocolitica infections in German federal states, 2001-2008. Data on the map shows mean annual incidence per 100,000 population by federal state. 
Table 2: Domestically acquired and imported Yersinia enterocolitica infections, 2001-2008

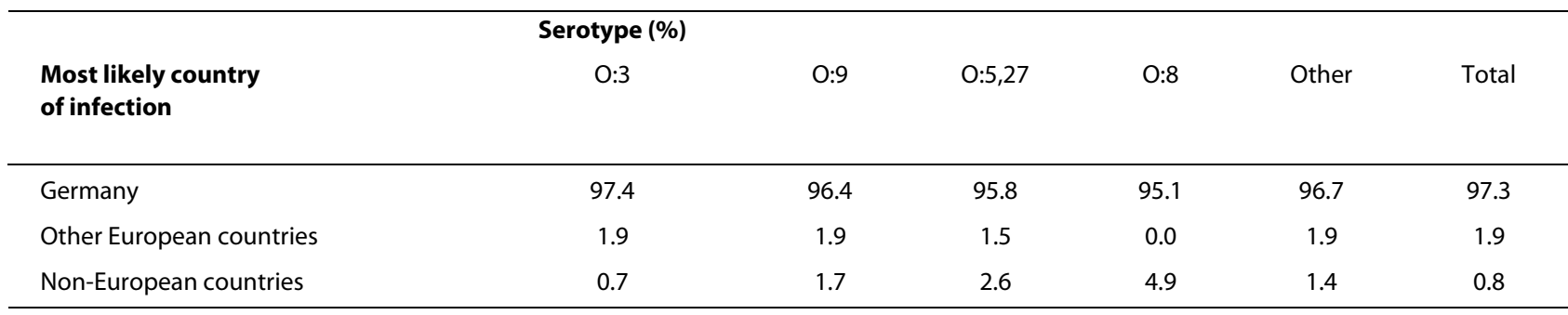

Reported frequency of country of infection is listed for different serotypes (as percentages). Total number of reported serotypes with available information on country of infection: $0: 3, n=30,513 ; 0: 9, n=2,006 ; 0: 5,27, n=265 ; 0: 8, n=61$; other serotypes, $n=1$,434. Category "other European countries" includes EU member states (without Germany) plus Iceland, Liechtenstein, Norway, and Switzerland.

nounced in the summer, contributing to the seasonal peak incidences associated with warmer months, as in illnesses caused by STEC and Campylobacter spp. [15,16].

Children were more frequently infected with $Y$. enterocolitica $\mathrm{O}: 3$ than adults, whereas adults $\geq 40$ years of age were more frequently infected by $Y$. enterocolitica serotype O:9 than younger age groups. Prior exposure of children to $Y$. enterocolitica O:3 may conceivably provide some protection against acute infections due to the same serotype later in life, but not necessarily from other serotypes. Hospitalization was reported for $17 \%$ of patients infected with $Y$. enterocolitica, which is lower than the proportion of hospitalizations among reported Salmonella spp. infections in Germany (24\%), but slightly higher than in reported Campylobacter spp. infections (14\%) (unpublished data). Hospitalization was longer than the median of 4 days when serotypes other than O:3 were diagnosed. Hospitalization rate was highest and length of hospital stay was twice as long as the median when infection was due to serotype O:8, which may support the findings that the course of disease is more severe with this serotype [25]. However, the total number of reported cases infected with serotype O:8 was low (62 reported cases over the study period) and insufficient for a more detailed analysis. Interestingly, the hospitalization rate was relatively high (28\%) among teenagers (10-19 years). Symptoms of yersiniosis can resemble symptoms of appendicitis in this age group (pseudoappendicitis), which may account for more frequent hospitalizations [26] and unnecessary appendectomies among teenagers [27].

In Germany, the trend in the number of reportable cases of yersiniosis has been downward since 2002. The reason for this is uncertain, but can be observed for other gastrointestinal infections caused by enteric pathogens, e.g., salmonellosis, as well [28], with the exception of Campylobacter spp. infections. Improved food safety control measures and better hygiene measures during food preparation at the consumer level are possible explanations for the continuing decrease of $Y$. enterocolitica infections. Compared to other European countries, the incidence of yersiniosis in Germany remains relatively

Table 3: Percentage of reported Yersinia enterocolitica serotypes according to age group, 2001-2008

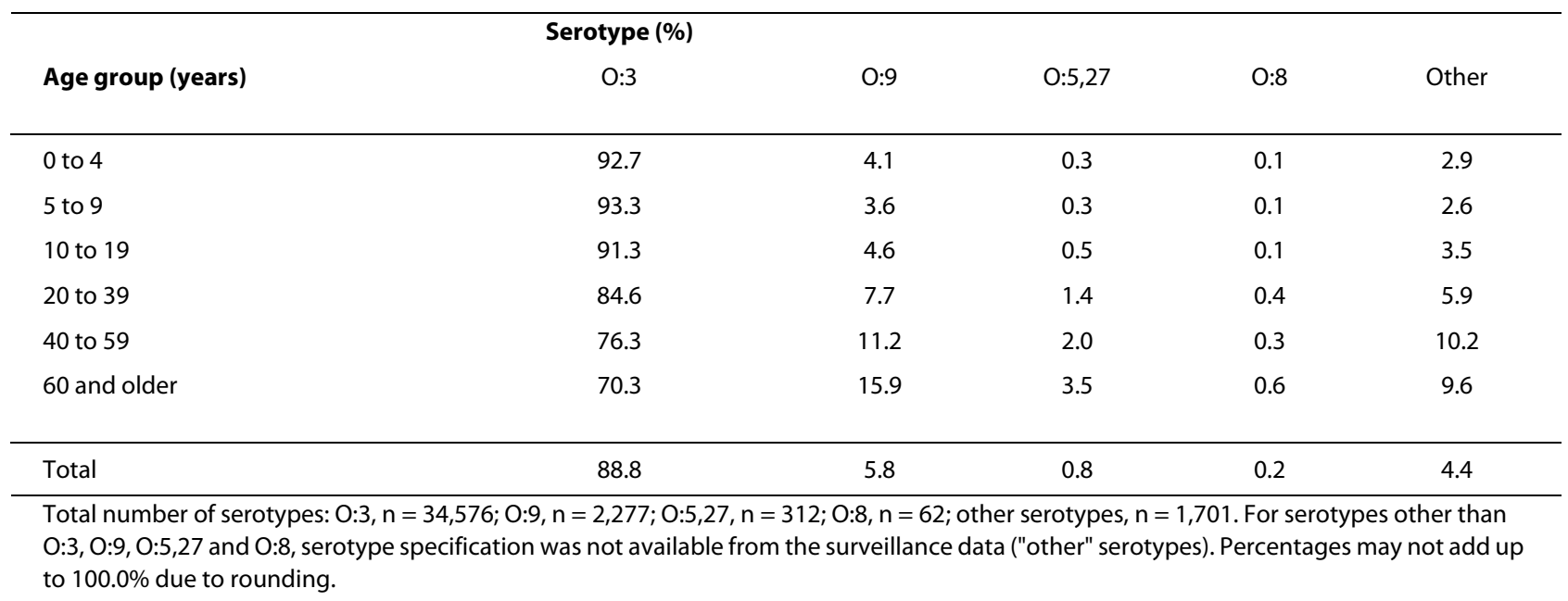


Table 4: Symptoms of Yersinia enterocolitica infections

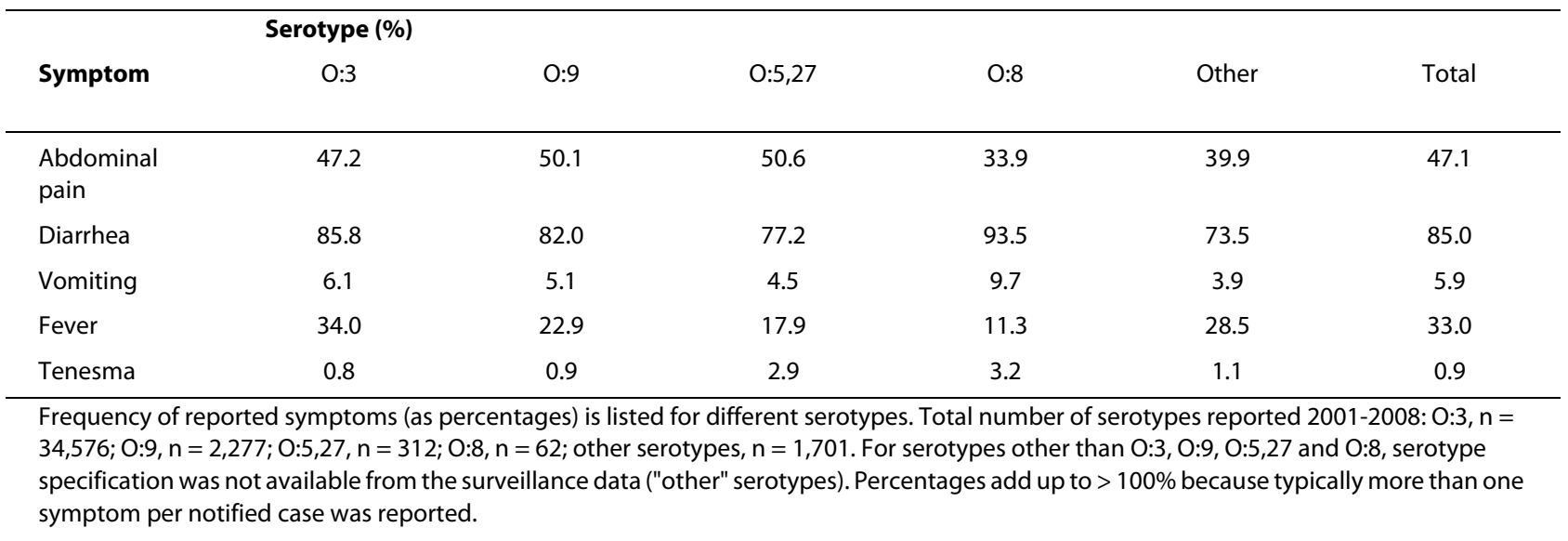

high. For example, in 2007, the overall incidence was about 2-fold higher (6.1/100,000 population) than the average in all European countries reporting to the European Centre for Disease Control and Prevention (ECDC) (2.9/100,000 population) [12]. Several reasons need to be considered: First, variability in reporting systems, frequency of diagnosis, and degree of underreporting among European countries may contribute to incidence differences. Second, since consumption of pork is a risk factor of yersiniosis, food preferences will play an important role. Pork is the most frequently consumed meat in Germany with an annual consumption of about $40 \mathrm{~kg}$ per capita [29]. Third, prevalence and concentration of $Y$. enterocolitica in food-producing animals and products made therefrom can also result in incidence differences among EU countries.

Surveillance data have their inherent limitations. For example, routine surveillance captures only a fraction of cases occurring in the population. Thus far, the degree of under-ascertainment remains to be systematically addressed in Germany. Based on studies that were conducted in other countries, it is estimated that for each culture-confirmed case of acute diarrheal illness, between 5 and 68 undiagnosed cases occur in the community $[22,30,31]$. Furthermore, a more severe course of disease is more likely to precipitate medical evaluation $[32,33]$, as is, probably, young age of the patient. Consequently, surveillance data are unlikely to be representative for the entirety of yersiniosis cases within the community. Surveillance data do not typically include detailed clinical information on every reported case, e.g., the presence of chronic diseases, cause of death, or detailed information on the laboratory diagnostic procedures. Despite these limitations, analysis of surveillance data can provide a good overview of the distribution of yersiniosis within the German population. However, identifying the risk factors of $Y$. enterocolitica infections, in particular among young children, requires analytical epidemiological methods. A case-control study is currently being conducted to elucidate and quantify the most important risk factors of $Y$. enterocolitica infections in Germany and assess sequelaeassociated risk factors, with the aim of recommending effective preventive measures that will improve disease control.

\section{Conclusions}

In Germany, yersiniosis is a zoonotic enteric disease with public health relevance because of its high incidence and the possible sequelae. Young children are affected most frequently, in particular one-year-old children, but incidence in this age group varies markedly from state to state. More research effort is required to elucidate risk factors of Yersinia enterocolitica infections, especially in young children.

Competing interests

The authors declare that they have no competing interests.

\section{Authors' contributions}

BR analyzed the data and wrote the manuscript. KS and DW critically reviewed the manuscript. All authors read and approved the final version.

\section{Acknowledgements}

This work was supported by grant $01 \mathrm{KI} 07127$ (Foodborne Zoonotic Infections of Humans; FBI-Zoo) from the German Federal Ministry of Education and Research (BMBF). The authors would like to thank Dr. Christina Frank for helpful comments on the manuscript.

\section{Author Details}

Robert Koch Institute, Department of Infectious Disease Epidemiology, DGZRing 1, 13086 Berlin, Germany

Received: 5 January 2010 Accepted: 14 June 2010

Published: 14 June 2010

\section{References}

1. Bottone EJ: Yersinia enterocolitica: overview and epidemiologic correlates. Microbes and Infection 1999, 1:323-333. 
2. Fosse J, Seegers $H$, Magras C: Prevalence and risk factors for bacterial food-borne zoonotic hazards in slaughter pigs: a review. Zoonoses Public Health 2009, 56:429-454

3. Fredriksson-Ahomaa M, Hallanvuo S, Korte T, Siitonen A, Korkeala H: Correspondence of genotypes of sporadic Yersinia enterocolitica bioserotype 4/O:3 strains from human and porcine sources. Epidemiol Infect 2001, 127:37-47.

4. Fredriksson-Ahomaa M, Stolle A, Siitonen A, Korkeala H: Sporadic human Yersinia enterocolitica infections caused by bioserotype 4/O:3 originate mainly from pigs. J Med Microbiol 2006, 55:747-749.

5. Tauxe RV, Wauters G, Goossens V, Van Noyen R, Vandepitte J, Martin SM, De Mol P, Thiers G: Yersinia enterocolitica infections and pork: the missing link. Lancet 1987:i:1129-1132.

6. Thompson JS, Gravel MJ: Family outbreak of gastroenteritis due to Yersinia enterocolitica serotype 0:3 from well water. Can J Microbiol 1986, 32(8):700-701.

7. Ostroff SM, Kapperud G, Hutwagner LC, Nesbakken T, Bean NH, Lassen J, Tauxe RV: Sources of sporadic Yersinia enterocolitica infections in Norway: a prospective case-control study. Epidemiol Infect 1994, 112:133-141.

8. Boqvist S, Pettersson H, Svensson Å, Andersson Y: Sources of sporadic Yersinia enterocolitica infection in children in Sweden, 2004: a casecontrol study. Epidemiol Infect 2008, 137(6):897-905.

9. Satterthwaite P, Pritchard K, Floyd D, Law B: A case-control study of Yersinia enterocolitica infections in Auckland. Austr NZ J Public Health 1999, 23:482-485

10. European Food|Safety Authority (EFSA): The Community Summary Report on Trends and Sources of Zoonoses and Zoonotic Agents in the European Union in 2007. EFSA J 2009:223.

11. Cover TL, Aber RC: Yersinia enterocolitica. NEng/ J Med 1989, 321:16-24.

12. European Centre for Disease Control and Prevention (ECDC): Annual Epidemiological Report on Communicable Diseases in Europe 2009. Stockholm: European Centre for Disease Prevention and Control; 2009

13. Faensen D, Claus H, Benzler J, Ammon A, Pfoch T, Breuer T, Krause G: SurvNet@RKI - a multistate electronic reporting system for communicable diseases. Euro Surveill 2006, 11(4): [http:// www.eurosurveillance.org/ViewArticle.aspx?Articleld=614].

14. Faensen D, Krause G: SurvStat@RKI - a web-based solution to query surveillance data in Germany. Euro Surveill 2004, 8(22): pii $=2477$

15. Werber D, Behnke S, Fruth A, Merle R, Menzler S, Glaser S, Kreienbrock L, Prager R, Tschäpe H, Roggentin P, Bockemühl J, Ammon A: Shiga toxinproducing Escherichia coli infection in Germany-different risk factors for different age groups. Am J Epidemiol 2007, 165:425-434.

16. Ethelberg S, Simonsen J, Gerner-Smidt G, Olsen KEP, Mølbak K: Spatial distribution and registry-based case-control analysis of Campylobacter infections in Denmark, 1991-2001. Am J Epidemio/ 2005, 162:1008-1015.

17. Robert Koch-Institut: Infektionsepidemiologisches Jahrbuch meldepflichtiger Krankheiten für 2008. Berlin: Robert Koch-Institut; 2009 .

18. Koehler KM, Lasky T, Fein SB, DeLong SM, Hawkins MA, Rabatsky-Ehr T, Ray SM, Shiferaw B, Swanson E, Vugia DJ, EIP FoodNet Working Group: Population-based incidence of infection with selected bacterial enteric pathogens in children younger than five years of age, 1996-1998. Ped Inf Dis J 2006, 25:129-134.

19. Verhaegen J, Charlier J, Lemmens P, Delmée M, Van Noyen R, Verbist L, Wauters G: Surveillance of human Yersinia enterocolitica infections in Belgium: 1967-1996. Clin Infect Dis 1998, 27:59-64.

20. Centers for Disease Control and Prevention: Preliminary Food Net data on the incidence of infection with pathogens transmitted commonly through food-10 States, 2008. MMWR Weekly 2009, 58(13):333-337.

21. Cohen MB: Etiology and mechanisms of acute infectious diarrhea in infants in the United States. J Pediatr 1991, 118:S34-S39.

22. Scallan E, Jones TF, Cronquist A, Thomas S, Frenzen P, Hoefer D, Medus C, Angulo FJ, The Foodnet Working Group: Factors associated with seeking medical care and submitting a stool sample in estimating the burden of foodborne illness. Foodborne Pathog Dis 2006, 3:432-438.

23. Held L, Graziano G, Frank C, Rue H: Joint spatial analysis of gastrointestinal infectious diseases. Stat Methods Med Res 2006, 15:465-480

24. Max Rubner-Institute, Federal Research Centre for Nutrition and Food: National Nutrition Survey II, part 2. 2008 [http://www.was-esse-ich.de/]
25. Bottone EJ: Yersinia enterocolitica: The charisma continues. Clin Microbiol Rev 1997, 10:257-276.

26. Perdikogianni C, Galanakis E, Michalakis M, Giannoussi E, Maraki S, Tselentis Y, Charissis G: Yersinia enterocolitica infection mimicking surgical conditions. Pediatr Surg Int 2006, 22:589-592.

27. Van Noyen R, Selderslaghs R, Bekaert J, Wauters G, Vandepitte J: Causative role of Yersinia and other enteric pathogens in the appendicular syndrome. Eur J Clin Microbiol Infect Dis 1991, 10:735-741.

28. Frank C, Käsbohrer A, Stark K, Werber D: Marked decrease in reporting incidence of salmonellosis driven by lower rates of Salmonella Enteritidis infections in Germany in 2008 - a continuing trend. Euro Surveill 2009, 14:1-2

29. Statistisches Bundesamt: Vom Erzeuger zum Verbraucher, Fleischversorgung in Deutschland, 2008. 2008 [https://wwwec.destatis.de/csp/shop/sfg/

bpm.html.cms.cBroker.cls?cmspath=struktur,vollanzeige.csp\&ID=1022244 ]. Wiesbaden: Statistisches Bundesamt

30. Voetsch AC, Van Gilder TJ, Angulo FJ, Farley MM, Shallow S, Marcus R, Cieslak PR, Deneen VC, Tauxe RV, Emerging Infections Program FoodNet Working Group: FoodNet estimate of the burden of illness caused by nontyphoidal Salmonella infections in the United States. Clin Infect Dis 2004, 38:S127-S134.

31. Wheeler JG, Sethi D, Cowden JM, Wall PG, Rodrigues LC, Tompkins DS, Hudson MJ, Roderick PJ, Infectious Intestinal Disease Study Executive: Study of infectious intestinal disease in England: rates in the community, presenting to general practice, and reported to national surveillance. BMJ 1999, 318:1046-1050.

32. De Wit MAS, Kortbeek LM, Koopmans MPG, De Jager CJ, Wannet WJB, Bartelds AIM, Van Duynhoven YTHP: A comparison of gastroenteritis in a general practice-based study and a community-based study. Epidemiol Infect 2001, 127:389-397.

33. Tam CC, Rodrigues LC, O'Brien SJ: The study of infectious intestinal disease in England: what risk factors for presentation to general practice tell us about potential for selection bias in case-control studies of reported cases of diarrhoea. Int J Epidemiol 2003, 32:99-105.

Pre-publication history

The pre-publication history for this paper can be accessed here: http://www.biomedcentral.com/1471-2458/10/337/prepub

doi: $10.1186 / 1471-2458-10-337$

Cite this article as: Rosner et al., Epidemiology of reported Yersinia enterocolitica infections in Germany, 2001-2008 BMC Public Health 2010, 10:337

\section{Submit your next manuscript to BioMed Centra and take full advantage of:}

- Convenient online submission

- Thorough peer review

- No space constraints or color figure charges

- Immediate publication on acceptance

- Inclusion in PubMed, CAS, Scopus and Google Scholar

- Research which is freely available for redistribution 